\title{
Publisher Correction: Estimation of the year-on-year volatility and the unpredictability of the United States energy system
}

Evan D. Sherwin (D), Max Henrion (D) and Inês M. L. Azevedo (D)

Correction to: Nature Energy https://doi.org/10.1038/s41560-018-0121-4, published online 26 March 2018.

In the version of this Analysis originally published, the key for the size frequency in Fig. 4 was erroneously switched, and should have read $5 \%$ for the small black dot, and $50 \%$ for the large black dot. This has now been amended.

Published online: 12 March 2019

https://doi.org/10.1038/s41560-019-0371-9 